\title{
Decomposition of Automorphisms of Certain Solvable Subalgebra of Symplectic Lie Algebra over Commutative Rings
}

\author{
Xing Tao Wang and Lei Zhang \\ Department of Mathematics, Harbin Institute of Technology, Harbin 150001, China \\ Correspondence should be addressed to Xing Tao Wang, xingtao@hope.hit.edu.cn
}

Received 26 March 2012; Accepted 26 April 2012

Academic Editor: Helge Holden

Copyright (C) 2012 X. T. Wang and L. Zhang. This is an open access article distributed under the Creative Commons Attribution License, which permits unrestricted use, distribution, and reproduction in any medium, provided the original work is properly cited.

Let $C_{l+1}(R)$ be the $2(l+1) \times 2(l+1)$ matrix symplectic Lie algebra over a commutative ring $R$ with 2 invertible. Then $\mathbf{t}_{l+1}^{(C)}(R)=\left\{\left(\begin{array}{cc}\bar{m}_{1} & \bar{m}_{2} \\ 0 & -\bar{m}_{1}^{T}\end{array}\right) \mid \bar{m}_{1}\right.$ is an $l+1$ upper triangular matrix, $\bar{m}_{2}^{T}=\bar{m}_{2}$, over $R\}$ is the solvable subalgebra of $C_{l+1}(R)$. In this paper, we give an explicit description of the automorphism group of $\mathbf{t}_{l+1}^{(C)}(R)$.

\section{Introduction}

Classical Lie algebras occupy an important place in matrix algebras. Let $R$ be a commutative ring $R$ with the identity 1 and $R^{*}$ the group of invertible elements in $R$. Let $M_{n}(R)$ be the $R$-algebra of $n$ by $n$ matrices over $R$ that has a structure of a Lie algebra over $R$ with bracket operation $[x, y]=x y-y x$ for any $x, y \in M_{n}(R)$. The symplectic Lie algebra

$$
C_{l+1}(R)=\left\{X \mid X\left(\begin{array}{cc}
0 & I_{l} \\
-I_{l} & 0
\end{array}\right)+\left(\begin{array}{cc}
0 & I_{l} \\
-I_{l} & 0
\end{array}\right) X^{T}=0, X \in M_{2 l+2}(R)\right\}
$$

is one of classical Lie algebras, where $T$ denotes the matrix transpose. It is easy to show that the following subalgebra of $C_{l+1}(R)$ such that

$$
\mathbf{t}_{l+1}^{(C)}(R)=\left\{\left(\begin{array}{cc}
\bar{m}_{1} & \bar{m}_{2} \\
0 & -\bar{m}_{1}^{T}
\end{array}\right) \mid \bar{m}_{1} \text { is an } l+1 \text { upper triangular matrix, } \bar{m}_{2}^{T}=\bar{m}_{2}\right\}
$$

is solvable. 
Let $e$ be the identity matrix in $M_{n}(R)$ and let $e_{i j}^{(n)}$ denotes the matrix in $M_{n}(R)$ all of whose entries are 0 , except the $(i, j)$ th entry which is 1 . Let

$$
\begin{gathered}
\alpha_{i, i+k}=e_{i, i+k}^{(n)}-e_{l+i+k+1, l+i+1}^{(n)}, \quad i=1, \ldots, l-k+1, k=0,1, \ldots, l \\
\gamma_{i, i+k}=e_{i, l+i+k+1}^{(n)}+e_{i+k, l+i+1}^{(n)}, \quad i=1, \ldots, l-k+1, k=1, \ldots, l, \\
\gamma_{i i}=e_{i, l+i+1}^{(n)}, \quad i=1, \ldots, l+1,
\end{gathered}
$$

where $n=2(l+1), l \geq 1$. For discussion latter, we rewrite $\mathbf{t}_{l+1}^{(C)}(R)$ as

$$
\mathbf{t}_{l+1}^{(C)}(R)=\sum_{k=0}^{l} \sum_{i=1}^{l-k+1} R \alpha_{i, i+k}+\sum_{k=0}^{l} \sum_{i=1}^{l-k+1} R \gamma_{i, i+k} .
$$

Automorphisms of associative algebras have been explored in many articles [1-8]. Encouraged by Doković [9] and Cao's [10] papers which described the automorphism groups of Lie algebra consisting of all upper triangular $n \times n$ matrices of trace 0 over a connected commutative ring and a commutative ring with $n$ invertible, respectively, in this paper we use similar techniques to those in [11] to prove that any automorphism $\psi$ of $\mathbf{t}_{l+1}^{(C)}(R)$ can be uniquely expressed as $\psi=\theta \lambda_{D}$, where $\theta$ and $\lambda_{D}$ are inner and diagonal automorphisms, respectively, for $l \geq 1$ and $R$ is a commutative ring with 2 invertible. We also give an explicit description of the remaining case $l=0$.

Theorem 1.1. For any automorphism $\psi$ of $\mathbf{t}_{l+1}^{(C)}(R)(l \geq 1)$ there are unique inner and diagonal automorphisms, $\theta$ and $\lambda_{D}$, respectively, of $\mathbf{t}_{l+1}^{(C)}(R)$ such that $\psi=\theta \lambda_{D}$.

Theorem 1.2. Let $\supset$ and $\Phi$ be the inner and diagonal automorphism groups, respectively. Then $\operatorname{Aut}\left(\mathbf{t}_{l+1}^{(C)}(R)\right)=\supset \ltimes \boldsymbol{\Phi}$, where Aut $\left(\mathbf{t}_{l+1}^{(C)}(R)\right)$ denotes the automorphism group of $\mathbf{t}_{l+1}^{(C)}(R)$.

\section{Preliminaries}

Let

$$
\begin{aligned}
& P_{n}=\left\{\alpha_{i, i+k} \mid i=1, \ldots, l-k+1, k=0,1, \ldots, l\right\}, \\
& W_{n}=\left\{\gamma_{i, i+k} \mid i=1, \ldots, l-k+1, k=0,1, \ldots, l\right\} .
\end{aligned}
$$

Then the set $P_{n} \cup W_{n}$ is a basis of $\mathbf{t}_{l+1}^{(C)}(R)$.

Lemma 2.1. Let $H_{n}$ be the set generated by the set $\left\{\alpha_{j j}, \alpha_{i, i+1}, \gamma_{l+1, l+1} \mid 1 \leq j \leq l+1,1 \leq i \leq l\right\}$, where $n=2(l+1)$. Then $H_{n}=\mathbf{t}_{l+1}^{(C)}(R)$. 
Proof. We only need to show that $\mathbf{t}_{l+1}^{(C)}(R) \subseteq H_{n}$. It is obvious that $\alpha_{i, i+k} \in H_{n}$, when $k=0,1$. When $k=2$, we have

$$
\begin{aligned}
\alpha_{i, i+2} & =e_{i, i+2}^{(n)}-e_{l+i+3, l+i+1}^{(n)} \\
& =\left[\left(e_{i, i+1}^{(n)}-e_{l+i+2, l+i+1}^{(n)}\right),\left(e_{i+1, i+2}^{(n)}-e_{l+i+3, l+i+2}^{(n)}\right)\right] \\
& =\left[\alpha_{i, i+1}, \alpha_{i+1, i+2}\right] \in H_{n} .
\end{aligned}
$$

Assume that $\alpha_{i, i+k-1} \in H_{n}$, then $\alpha_{i, i+k}=\left[\alpha_{i, i+k-1}, \alpha_{i+k-1, i+k}\right] \in H_{n}$, that is, $P_{n} \subseteq H_{n}$. Since $\gamma_{l+1, l+1} \in$ $H_{n}$, for any $\gamma_{l-k+1, l-k+2} \in W_{n}$, when $k=1$,

$$
\begin{aligned}
\gamma_{l, l+1} & =e_{l, 2 l+2}^{(n)}+e_{l+1,2 l+1}^{(n)} \\
& =\left[\left(e_{l, l+1}^{(n)}-e_{2 l+2,2 l+1}^{(n)}\right), e_{l+1,2 l+2}^{(n)}\right] \\
& =\left[\alpha_{l, l+1}, \gamma_{l+1, l+1}\right] \in H_{n} .
\end{aligned}
$$

Assume that when $k=m-1, \gamma_{l-m+2, l-m+3} \in H_{n}$, then when $k=m, \gamma_{l-m+1, l-m+2}=\left[\alpha_{l-m+1, l-m+3}\right.$, $\left.\gamma_{l-m+2, l-m+3}\right] \in H_{n}$, that is, $\gamma_{i, i+1} \in H_{n}, i=1, \ldots, l$. For any $\gamma_{i, i+k} \in H_{n}(k \geq 1)$, when $k=1$, $\gamma_{i, i+1} \in H_{n}$. When $k \geq 2, \gamma_{i, i+k}=\left[\alpha_{i, i+k-1}, \gamma_{i+k-1, i+k}\right] \in H_{n}$. Since $2 \gamma_{i i}=\left[\alpha_{i, i+1}, \gamma_{i, i+1}\right] \in H_{n}$, and 2 is invertible, we have $\gamma_{i i} \in H_{n}$ for $1 \leq i \leq l$. Thus $W_{n} \subseteq H_{n}$. Because $P_{n} \cup W_{n}$ is a basis of $\mathbf{t}_{l+1}^{(C)}(R)$, we obtain $\mathbf{t}_{l+1}^{(C)}(R) \subseteq H_{n}$.

Now, denote $\mathbf{t}_{l+1}^{(C)}(R)$ by $\mathbf{n}_{0}^{(C)}$. Let $\mathbf{n}_{1}^{(C)}=\left[\mathbf{n}_{0}^{(C)}, \mathbf{n}_{0}^{(C)}\right], \mathbf{n}_{2}^{(C)}=\left[\mathbf{n}_{1}^{(C)}, \mathbf{n}_{1}^{(C)}\right], \mathbf{n}_{j}^{(C)}=\left[\mathbf{n}_{1}^{(C)}\right.$, $\left.\mathbf{n}_{j-1}^{(C)}\right], j=3, \ldots, 2 l+1$. It is not difficult to know

$$
\begin{gathered}
\mathbf{n}_{j}^{(C)}=\sum_{k=j}^{l} \sum_{i=1}^{l-k+1} R \alpha_{i, i+k}+\sum_{k=j}^{l} \sum_{i=l+2-[(k+1) / 2]}^{l+1} R \gamma_{2 l-k-i+3, i} \\
+\sum_{k=l+1}^{2 l+1} \sum_{i=1}^{l+1-[k / 2]} R \gamma_{i, 2 l-k-i+3,} \quad 1 \leq j \leq l, \\
\mathbf{n}_{j}^{(C)}=\sum_{k=j}^{2 l+1} \sum_{i=1}^{l+1-[k / 2]} R \gamma_{i, 2 l-k-i+3,} \quad l+1 \leq j \leq 2 l+1(l \geq 2), \\
\mathbf{n}_{j}^{(C)}=0, \quad 2 l+2 \leq j .
\end{gathered}
$$

It is easy to check that $\left[\mathbf{n}_{m}^{(C)}, \mathbf{n}_{l}^{(C)}\right] \subseteq \mathbf{n}_{m+l}^{(C)}$ for $m+l \leq 2 l+1$ or $\left[\mathbf{n}_{m}^{(C)}, \mathbf{n}_{l}^{(C)}\right]=0$ for $m+l \geq$ $2 l+2$. For any $\psi \in \operatorname{Aut}\left(\mathbf{n}_{0}^{(C)}\right)$, we have $\psi\left(\mathbf{n}_{1}^{(C)}\right)=\left[\psi\left(\mathbf{n}_{0}^{(C)}\right), \psi\left(\mathbf{n}_{0}^{(C)}\right)\right]=\left[\mathbf{n}_{0}^{(C)}, \mathbf{n}_{0}^{(C)}\right]=\mathbf{n}_{1}^{(C)}$ and $\psi\left(\mathbf{n}_{j}^{(C)}\right)=\mathbf{n}_{j}^{(C)}, j=2, \ldots, 2 l+1$. Therefore, $\psi\left(\mathbf{n}_{j-1}^{(C)} \backslash \mathbf{n}_{j}^{(C)}\right)=\mathbf{n}_{j-1}^{(C)} \backslash \mathbf{n}_{j}^{(C)}, j=1, \ldots, 2 l+1$. Note that if $\gamma_{i j} \in W_{n}$, then $\gamma_{i j} \in \mathbf{n}_{2 l-i-j+3}^{(C)} \backslash \mathbf{n}_{2 l-i-j+4}^{(C)}$.

For any maximal ideal $M$ of $R, \bar{R}=R / M$ is a field. The natural homomorphism $\pi$ : $R \rightarrow \bar{R}$ induces a homomorphism $\psi_{M}: \mathbf{t}_{l+1}^{(C)}(R) \rightarrow \mathbf{t}_{l+1}^{(C)}(\bar{R})$ which is surjective. So every 
automorphism $\psi$ of $\mathbf{t}_{l+1}^{(C)}(R)$ may induce an automorphism $\bar{\psi}$ of $\mathbf{t}_{l+1}^{(C)}(\bar{R})$. Using this fact and that $\mathbf{n}_{2 l+1}^{(C)}=R \gamma_{11}$ (for $l \geq 1$ ), we have that $\psi\left(\gamma_{11}\right)=c_{11} \gamma_{11}$, where $c_{11} \in R^{*}$. Otherwise, $c_{11}$ should be contained in a maximal ideal $M$ of $R$, then $\bar{\psi}\left(\bar{\gamma}_{11}\right)=0$ on $\mathbf{t}_{l+1}^{(C)}(\bar{R})$, where $\bar{\gamma}_{11}$ is the image of $\gamma_{11}$ in $\mathbf{t}_{l+1}^{(C)}(\bar{R})$, which is impossible.

Lemma 2.2. Let $\psi$ be in $\operatorname{Aut}\left(\mathbf{n}_{0}^{(C)}\right)$. If $\psi\left(\alpha_{j j}\right), \psi\left(\alpha_{j, j+1}\right)$ and $\psi\left(\gamma_{l+1, l+1}\right)$ are expressed, respectively, as

$$
\begin{gathered}
\psi\left(\alpha_{j j}\right)=\sum_{i=1}^{l+1} a_{i i}^{(j)} \alpha_{i i} \bmod \mathbf{n}_{1}^{(C)}, j=1, \ldots, l+1, \\
\psi\left(\alpha_{j, j+1}\right)=\sum_{i=1}^{l} \tilde{a}_{i, i+1}^{(j)} \alpha_{i, i+1}+\widetilde{c}_{l+1, l+1}^{(j)} \gamma_{l+1, l+1} \bmod \mathbf{n}_{2}^{(C)}, j=1, \ldots, l, \\
\psi\left(\gamma_{l+1, l+1}\right)=\sum_{i=1}^{l} \widehat{a}_{i, i+1}^{(l+1)} \alpha_{i, i+1}+\widehat{c}_{l+1, l+1}^{(l+1)} \gamma_{l+1, l+1} \bmod \mathbf{n}_{2}^{(C)},
\end{gathered}
$$

then the following matrices are invertible.

(i) $A=\left(a_{j i}\right)_{(l+1) \times(l+1)^{\prime}}$ where $a_{j i}=a_{i i}^{(j)}, j=1, \ldots, l+1, i=1, \ldots, l+1$;

(ii) $B=\left(b_{j i}\right)_{(l+1) \times(l+1)}$, where $b_{j i}=\tilde{a}_{i, i+1}^{(j)}, j=1, \ldots, l, i=1, \ldots, l, b_{j, l+1}=\tilde{c}_{l+1, l+1}^{(j)}, j=1, \ldots, l$, $b_{l+1, i}=\widehat{a}_{i, i+1}^{(l+1)}, i=1, \ldots, l$ and $b_{l+1, l+1}=\widehat{c}_{l+1, l+1}^{(l+1)}$.

Proof. (i) That $A$ is invertible follows from the fact that $\psi$ induces an automorphism of the free $R$-module $\mathbf{n}_{0}^{(C)} / \mathbf{n}_{1}^{(C)}$ of rank $l+1$ on the basis $\left\{\alpha_{j j}+\mathbf{n}_{1}^{(C)} \mid j=1, \ldots, l+1\right\}$. (ii) Note that $\psi$ induces an automorphism of the free $R$-module $\mathbf{n}_{1}^{(\mathrm{C})} / \mathbf{n}_{2}^{(\mathrm{C})}$ of rank $l+1$ on the basis $\left\{\alpha_{j, j+1}+\right.$ $\left.\mathbf{n}_{2}^{(C)}, \gamma_{l+1, l+1}+\mathbf{n}_{2}^{(C)} \mid j=1, \ldots, l\right\}$.

Lemma 2.3. Let $\psi \in \operatorname{Aut}\left(\mathbf{n}_{0}^{(C)}\right)(l \geq 2)$. Write $\psi\left(\alpha_{j j}\right), \psi\left(\alpha_{j, j+1}\right)$, and $\psi\left(\gamma_{l+1, l+1}\right)$ as in (2.5)-(2.7), respectively. Then the following conclusions hold.

(i) For $1 \leq m, k, h \leq l, \tilde{a}_{h, h+1}^{(m)} \tilde{a}_{k, k+1}^{(m)}=0(h \neq k), \tilde{a}_{h, h+1}^{(m)} \widetilde{c}_{l+1, l+1}^{(m)}=0, \widehat{a}_{h, h+1}^{(l+1)} \widehat{a}_{k, k+1}^{(l+1)}=0(h \neq k)$ and $\widehat{a}_{h, h+1}^{(l+1)} \widehat{c}_{l+1, l+1}^{(l+1)}=0$.

(ii) For $1 \leq k, h \leq l,\left(a_{h h}^{(i)}-a_{h+1, h+1}^{(i)}\right)\left(a_{k k}^{(i)}-a_{k+1, k+1}^{(i)}\right)=0(h \neq k)$ and $\left(a_{h h}^{(i)}-a_{h+1, h+1}^{(i)}\right) a_{l+1, l+1}^{(i)}=0$ $(1 \leq h \leq l$, here $l \geq 1)$, where $i=1, l+1$.

(iii) For $2 \leq m \leq l$ and $1 \leq i, k, h \leq l,\left(a_{i i}^{(m)}-a_{i+1, i+1}^{(m)}\right)\left(a_{h h}^{(m)}-a_{h+1, h+1}^{(m)}\right)\left(a_{k k}^{(m)}-a_{k+1, k+1}^{(m)}\right)=0$ $(i \neq h \neq k \neq i$, here $l \geq 3)$ and $\left(a_{i i}^{(m)}-a_{i+1, i+1}^{(m)}\right)\left(a_{h h}^{(m)}-a_{h+1, h+1}^{(m)}\right) a_{l+1, l+1}^{(m)}=0(1 \leq i \neq h \leq l)$.

Proof. (i) When $j \neq m, m+1,\left[\psi\left(\alpha_{j j}\right), \psi\left(\alpha_{m, m+1}\right)\right]=0$. So

$$
\tilde{a}_{i, i+1}^{(m)}\left(a_{i i}^{(j)}-a_{i+1, i+1}^{(j)}\right)=0, \quad \widetilde{c}_{l+1, l+1}^{(m)} a_{l+1, l+1}^{(j)}=0 .
$$


From $\left[\psi\left(\alpha_{m m}\right), \psi\left(\alpha_{m, m+1}\right)\right]=\psi\left(\alpha_{m, m+1}\right)$ and $\left[\psi\left(\alpha_{m+1, m+1}\right), \psi\left(\alpha_{m, m+1}\right)\right]=-\psi\left(\alpha_{m, m+1}\right)$, we have

$$
\begin{array}{cc}
\tilde{a}_{i, i+1}^{(m)}\left(a_{i i}^{(m)}-a_{i+1, i+1}^{(m)}\right)=\tilde{a}_{i, i+1}^{(m)}, \quad 2 \widetilde{c}_{l+1, l+1}^{(m)} a_{l+1, l+1}^{(m)}=\widetilde{c}_{l+1, l+1}^{(m)} \\
\tilde{a}_{i, i+1}^{(m)}\left(a_{i i}^{(m+1)}-a_{i+1, i+1}^{(m+1)}\right)=-\tilde{a}_{i, i+1}^{(m)}, \quad 2 \widetilde{c}_{l+1, l+1}^{(m)} a_{l+1, l+1}^{(m+1)}=-\widetilde{c}_{l+1, l+1}^{(m)} .
\end{array}
$$

When $j \neq l+1,\left[\psi\left(\alpha_{j j}\right), \psi\left(\gamma_{l+1, l+1}\right)\right]=0$. So

$$
\widehat{a}_{i, i+1}^{(l+1)}\left(a_{i i}^{(j)}-a_{i+1, i+1}^{(j)}\right)=0, \quad \widehat{c}_{l+1, l+1}^{(l+1)} a_{l+1, l+1}^{(j)}=0 .
$$

From $\left[\psi\left(\alpha_{l+1, l+1}\right), \psi\left(\gamma_{l+1, l+1}\right)\right]=2 \psi\left(\gamma_{l+1, l+1}\right)$, we have

$$
\widehat{a}_{i, i+1}^{(l+1)}\left(a_{i i}^{(l+1)}-a_{i+1, i+1}^{(l+1)}\right)=2 \widehat{a}_{i, i+1}^{(l+1)}, \quad \widehat{c}_{l+1, l+1}^{(l+1)} a_{l+1, l+1}^{(l+1)}=\widehat{c}_{l+1, l+1}^{(l+1)} .
$$

Let $C=\left(c_{j i}\right)_{(l+1) \times(l+1)}$, where $c_{j i}=a_{i i}^{(j)}-a_{i+1, i+1}^{(j)}, j=1, \ldots, l+1, i=1, \ldots, l$, and $c_{j, l+1}=2 a_{l+1, l+1}^{(j)}$, $j=1, \ldots, l+1$. By Lemma $2.2, \operatorname{det} A \in R^{*}$, so $\operatorname{det} C \in R^{*}$. Investigating $\tilde{a}_{h, h+1}^{(m)} \tilde{a}_{k, k+1}^{(m)} \operatorname{det} C$, we may find that $h$ th column and $k$ th column are linearly dependent (both are the form $\left.\left(0, \ldots, 0, \tilde{a}_{j, j+1}^{(m)},-\tilde{a}_{j, j+1}^{(m)}, 0, \ldots, 0\right)^{t}, j=h, k\right)$ by $(2.6)$ and $(2.7)$, so $\tilde{a}_{h, h+1}^{(m)} \tilde{a}_{k, k+1}^{(m)} \operatorname{det} C=0$. Similarly, $\tilde{a}_{h, h+1}^{(m)} \widetilde{c}_{l+1, l+1}^{(m)} \operatorname{det} C=0, \widehat{a}_{h, h+1}^{(l+1)} \widehat{a}_{k, k+1}^{(l+1)} \operatorname{det} C=0(h \neq k)$ and $\widehat{a}_{h, h+1}^{(l+1)} \widehat{c}_{l+1, l+1}^{(l+1)} \operatorname{det} C=0$. Then $\widetilde{a}_{h, h+1}^{(m)}$ $\tilde{a}_{k, k+1}^{(m)}=0(h \neq k), \tilde{a}_{h, h+1}^{(m)} \tilde{c}_{l+1, l+1}^{(m)}=0, \widehat{a}_{h, h+1}^{(l+1)} \widehat{a}_{k, k+1}^{(l+1)}=0(h \neq k)$, and $\widehat{a}_{h, h+1}^{(l+1)} \widehat{c}_{l+1, l+1}^{(l+1)}=0$.

(ii) When $i=1$, from $\left(a_{h h}^{(1)}-a_{h+1, h+1}^{(1)}\right)\left(a_{k k}^{(1)}-a_{k+1, k+1}^{(1)}\right) \operatorname{det} B=0(h \neq k)$, we have $\left(a_{h h}^{(1)}-\right.$ $\left.a_{h+1, h+1}^{(1)}\right)\left(a_{k k}^{(1)}-a_{k+1, k+1}^{(1)}\right)=0(h \neq k)$. Similarly, we have $\left(a_{h h}^{(1)}-a_{h+1, h+1}^{(1)}\right)\left(a_{l l}^{(1)}+a_{l+1, l+1}^{(1)}\right)=0(1 \leq$ $h \leq l)$. When $i=l+1$, we get the results similarly.

(iii) The proving process is similar to (i) and (ii).

Lemma 2.4. Let $\psi \in \operatorname{Aut}\left(\mathbf{n}_{0}^{(C)}\right)$. Then

(i) when $l \geq 1, \psi\left(\alpha_{12}\right)=\tilde{a}_{12}^{(1)} \alpha_{12} \bmod \mathbf{n}_{2}^{(C)}$, where $\tilde{a}_{12}^{(1)} \in R^{*}$;

(ii) if $\psi\left(\alpha_{12}\right)=\tilde{a}_{12}^{(1)} \alpha_{12} \bmod \mathbf{n}_{2}^{(C)}$, where $\tilde{a}_{12}^{(1)} \in R^{*}$, then $\psi\left(\alpha_{i, i+1}\right)=\tilde{a}_{i, i+1}^{(i)} \alpha_{i, i+1} \bmod \mathbf{n}_{2}^{(C)}$ and $\psi\left(\gamma_{l+1, l+1}\right)=\widehat{c}_{l+1, l+1}^{(l+1)} \gamma_{l+1, l+1} \bmod \mathbf{n}_{2}^{(C)}$, where $\tilde{a}_{i, i+1}^{(i)}, \widehat{c}_{l+1, l+1}^{(l+1)} \in R^{*}$.

Proof. (i) Noting that $\alpha_{i, i+1}, \gamma_{l+1, l+1} \in \mathbf{n}_{1}^{(C)} \backslash \mathbf{n}_{2}^{(C)}$ and $\gamma_{12} \in \mathbf{n}_{2 l}^{(C)} \backslash \mathbf{n}_{2 l+1}^{(C)}$, we have $\psi\left(\alpha_{12}\right) \in \mathbf{n}_{1}^{(C)} \backslash \mathbf{n}_{2}^{(C)}$ and $\psi\left(\gamma_{12}\right)=\widehat{c}_{12}^{(12)} \gamma_{12} \bmod \mathbf{n}_{2 l+1}^{(C)} \in \mathbf{n}_{2 l}^{(C)} \backslash \mathbf{n}_{2 l+1}^{(C)}$, where $\widehat{c}_{12}^{(12)} \in R^{*}$. Using (2.7), from $\left[\psi\left(\alpha_{11}\right)\right.$, $\left.\psi\left(\gamma_{12}\right)\right]=\psi\left(\gamma_{12}\right)$, we have $\widehat{c}_{12}^{(12)}\left(a_{11}^{(1)}+a_{22}^{(1)}\right)=\widehat{c}_{12}^{(12)}$, that is, $a_{11}^{(1)}+a_{22}^{(1)}=1$. Write $\psi\left(\alpha_{11}\right)$ and $\psi\left(\gamma_{11}\right)$ as $\psi\left(\alpha_{11}\right)=\sum_{i=1}^{l+1} a_{i i}^{(1)} \alpha_{i i} \bmod \mathbf{n}_{1}^{(C)}$ and $\psi\left(\gamma_{11}\right)=c_{11}^{*} \gamma_{11} \in \mathbf{n}_{2 l+1}^{(C)}$, where $c_{11}^{*} \in R^{*}$. From $2 \psi\left(\gamma_{11}\right)=$ $\left[\psi\left(\alpha_{11}\right), \psi\left(\gamma_{11}\right)\right]=2 a_{11}^{(1)} c_{11}^{*} \gamma_{11}$, we have $a_{11}^{(1)}=1$. Then $a_{22}^{(1)}=0$. By Lemma 2.3 we have $a_{i i}^{(1)}-$ $a_{i+1, i+1}^{(1)}=0, i=2, \ldots, l$ (here $l \geq 2$ ) and $a_{l+1, l+1}^{(1)}=0$. So $a_{i i}^{(1)}=0, i=2, \ldots, l+1$, that is, $\psi\left(\alpha_{11}\right)=$ $a_{11}^{(1)} \bmod \mathbf{n}_{1}^{(C)}$. Then $\psi\left(\alpha_{12}\right)=\left[\psi\left(\alpha_{11}\right), \psi\left(\alpha_{12}\right)\right]=\tilde{a}_{12}^{(1)} \alpha_{12} \bmod \mathbf{n}_{2}^{(C)}$ and $\tilde{a}_{12}^{(1)} \in R^{*}$. By Lemma 2.3, (i) holds. 
(ii) Write $\psi\left(\alpha_{j, j+1}\right)$ and $\psi\left(\gamma_{l+1, l+1}\right)$ as (2.6) and (2.7), respectively. From $\psi\left(\alpha_{13}\right)=\left[\psi\left(\alpha_{12}\right)\right.$, $\left.\psi\left(\alpha_{23}\right)\right]$, we have $\psi\left(\alpha_{13}\right)=\tilde{a}_{12}^{(1)} \tilde{a}_{23}^{(2)} \alpha_{13} \bmod \mathbf{n}_{3}^{(C)}$. Since $\alpha_{13} \in \mathbf{n}_{2}^{(C)} \backslash \mathbf{n}_{3}^{(C)}, \psi\left(\alpha_{13}\right) \in \mathbf{n}_{2}^{(C)} \backslash \mathbf{n}_{3}^{(C)}$. So $\tilde{a}_{12}^{(1)} \tilde{a}_{23}^{(2)} \in R^{*}$, that is, $\tilde{a}_{23}^{(2)} \in R^{*}$. In general, for $m=2, \ldots, l$, we have

$$
\begin{gathered}
\psi\left(\alpha_{1, m+1}\right)=\prod_{i=1}^{m} \tilde{a}_{i, i+1}^{(i)} \alpha_{1, m+1} \bmod \mathbf{n}_{m+1}^{(C)} \in \mathbf{n}_{m}^{(C)} \backslash \mathbf{n}_{m+1}^{(C)}, \quad m=1, \ldots, l \\
\psi\left(\gamma_{1, l+1}\right)=\left[\psi\left(\alpha_{1, l+1}\right), \psi\left(\gamma_{l+1, l+1}\right)\right]=\prod_{i=1}^{l} \tilde{a}_{i, i+1}^{(i)} \widehat{c}_{l+1, l+1}^{(l+1)} \gamma_{1, l+1} \bmod \mathbf{n}_{l+2}^{(C)} \in \mathbf{n}_{l+1}^{(C)} \backslash \mathbf{n}_{l+2}^{(C)},
\end{gathered}
$$

here $\tilde{a}_{i, i+1}^{(i)}, \widehat{c}_{l+1, l+1}^{(l+1)}$ should be in $R^{*}, i=1, \ldots, l$. By Lemma 2.3 we have that $\tilde{a}_{i, i+1}^{(j)}=0(i \neq j)$, $\widetilde{c}_{l+1, l+1}^{(j)}=0, j=1, \ldots, l$, and $\hat{a}_{i, i+1}^{(l+1)}, i=1, \ldots, l$. Hence $\psi\left(\alpha_{l, l+1}\right)$ and $\psi\left(\gamma_{l+1, l+1}\right)$ have the required forms, respectively.

\section{The Standard Automorphisms of $\mathbf{t}_{l+1}^{(C)}(R)$}

Now let us introduce two types of Lie automorphisms of $\mathbf{t}_{l+1}^{(C)}(R)$.

\section{(i) Inner Automorphisms}

Let $r=I_{n}+a \alpha_{i j}(i \neq j)$ or $r=I_{n}+a \gamma_{i j}$. It is easy to check that $r y r^{-1} \in \mathbf{n}_{0}^{(C)}$. The map $\theta_{r}: \mathbf{t}_{l+1}^{(C)}(R) \rightarrow$ $\mathbf{t}_{l+1}^{(C)}(R)$ such that $x \mapsto r x r^{-1}, x \in \mathbf{n}_{0}^{(C)}$, defines an automorphism of $\mathbf{n}_{0}^{(C)}$, which is called an inner automorphism (note that $r$ is a symplectic matrix defined by $\left(\begin{array}{cc}0 & I_{I_{1+1}} \\ -I_{l+1} & 0\end{array}\right)$ ). We denote $\theta_{r}$ by $\theta_{a a_{i j}}, \theta_{a \gamma_{i j}}$, respectively. In these cases, we have $\theta_{a \alpha_{i j}}^{-1}=\theta_{-a a_{i j}}, \theta_{a \gamma_{i j}}^{-1}=\theta_{-a \gamma_{i j}}$, respectively, and that $\theta_{a \alpha_{i j}}\left(\alpha_{i i}\right)=\alpha_{i i}-a \alpha_{i j}, \theta_{a \alpha_{i j}}\left(\alpha_{j j}\right)=\alpha_{j j}+a \alpha_{i j}, \theta_{a \alpha_{i j}}\left(\alpha_{k k}\right)=\alpha_{k k}(k \neq i, j), \theta_{a \alpha_{i j}}\left(\alpha_{k, k+1}\right)=$ $\alpha_{k, k+1}(k \neq j, i-1), \theta_{a \alpha_{i j}}\left(\alpha_{j, j+1}\right)=\alpha_{j, j+1}+a \alpha_{i, j+1}, \theta_{a \alpha_{i j}}\left(\alpha_{i-1, i}\right)=\alpha_{i-1, i}-a \alpha_{i-1, j}, \theta_{a \gamma_{i i}}\left(\alpha_{i i}\right)=\alpha_{i i}-2 a \gamma_{i i}$, $\theta_{a \gamma_{i, i+1}}\left(\alpha_{i, i+1}\right)=\alpha_{i, i+1}-2 a \gamma_{i i}$ and $\theta_{a \alpha_{i, i+1}}\left(\gamma_{i, i+1}\right)=\gamma_{i, i+1}+2 a \gamma_{i i}$. All inner automorphisms of $\mathbf{t}_{l+1}^{(C)}(R)$ generate a subgroup of $\operatorname{Aut}\left(\mathbf{n}_{0}^{(C)}\right)$, which is denoted by $\supset$.

\section{(ii) Diagonal Automorphisms}

Let $d_{i} \in R^{*}, i=0,1, \ldots, l+1, d=\operatorname{diag}\left(d_{1}, \ldots, d_{l+1}\right)$ and $D=\operatorname{diag}\left(d, d^{-1} d_{0}\right)$. The map $\lambda_{D}$ : $\mathbf{t}_{l+1}^{(C)}(R) \rightarrow \mathbf{t}_{l+1}^{(C)}(R)$ such that $x \mapsto D x D^{-1}, x \in \mathbf{n}_{0}^{(C)}$, defines an automorphism of $\mathbf{t}_{l+1}^{(C)}(R)$, which is called a diagonal automorphism. It is clear that $\lambda_{D} \lambda_{\bar{D}}=\lambda_{D \bar{D}}$. So the set of diagonal automorphisms of $\mathbf{t}_{l+1}^{(C)}(R)$ is a subgroup of $\operatorname{Aut}\left(\mathbf{n}_{0}^{(C)}\right)$, which is denoted by $\boldsymbol{\Phi}$.

\section{Lemmas for Main Results}

Lemma 4.1. Let $\psi \in \operatorname{Aut}\left(\mathbf{n}_{0}^{(C)}\right)$. The following two statements are equivalent:

(i) $\psi\left(\alpha_{j, j+1}\right)=\tilde{a}_{j, j+1}^{(j)} \alpha_{j, j+1} \bmod \mathbf{n}_{2}^{(C)}$ and $\psi\left(\gamma_{l+1, l+1}\right)=\widehat{c}_{l+1, l+1}^{(l+1)} \gamma_{l+1, l+1} \bmod \mathbf{n}_{2}^{(C)}$, where $\tilde{a}_{j, j+1}^{(j)}$, $\widehat{c}_{l+1, l+1}^{(l+1)} \in R^{*}, j=1, \ldots, l ;$

(ii) $\psi\left(\alpha_{j j}\right)=\alpha_{j j} \bmod \mathbf{n}_{1}^{(C)}, j=1, \ldots, l+1$. 
Proof. (i) $\Rightarrow$ (ii). Write $\psi\left(\alpha_{j j}\right)$ as in (2.5). By the process of proving Lemma 2.3, we have $\tilde{a}_{12}^{(1)}$ $\left(a_{11}^{(1)}-a_{22}^{(1)}\right)=\tilde{a}_{12}^{(1)}, \tilde{a}_{i, i+1}^{(i)}\left(a_{i i}^{(i+1)}-a_{i+1, i+1}^{(i+1)}\right)=-\tilde{a}_{i, i+1}^{(i)}, \tilde{a}_{i+1, i+2}^{(i+1)}\left(a_{i+1, i+1}^{(i+1)}-a_{i+2, i+2}^{(i+1)}\right)=\tilde{a}_{i+1, i+2}^{(i+1)}, i=1, \ldots, l-1$ and $\tilde{a}_{l, l+1}^{(l)}\left(a_{l l}^{(l+1)}-a_{l+1, l+1}^{(l+1)}\right)=-\tilde{a}_{l, l+1}^{(l)}, \widehat{c}_{l+1, l+1}^{(l+1)} a_{l+1, l+1}^{(l+1)}=\widehat{c}_{l+1, l+1}^{(l+1)}$. Then we obtain that $a_{11}^{(1)}-a_{22}^{(1)}=1$, $a_{i i}^{(i+1)}-a_{i+1, i+1}^{(i+1)}=-1, a_{i+1, i+1}^{(i+1)}-a_{i+2, i+2}^{(i+1)}=1, i=1, \ldots, l-1$ and $a_{l+1, l+1}^{(l+1)}=1$. By Lemma 2.3 , we have $a_{j j}^{(j)}=1(1 \leq j \leq l+1)$ and $a_{i i}^{(j)}=0(i \neq j)$.

(ii) $\Rightarrow(\mathrm{i})$. Write $\psi\left(\alpha_{j, j+1}\right)$ and $\psi\left(\gamma_{l+1, l+1}\right)$, respectively, as in (2.6) and (2.7). Then

$$
\begin{gathered}
\psi\left(\alpha_{j, j+1}\right)=\left[\psi\left(\alpha_{j j}\right), \psi\left(\alpha_{j, j+1}\right)\right]=\tilde{a}_{j, j+1}^{(j)} \alpha_{j, j+1} \bmod \mathbf{n}_{2}^{(C)}, \quad j=1, \ldots, l, \\
2 \psi\left(\gamma_{l+1, l+1}\right)=\left[\psi\left(\alpha_{l+1, l+1}\right), \psi\left(\gamma_{l+1, l+1}\right)\right]=2 \widehat{c}_{l+1, l+1}^{(l+1)} \gamma_{l+1, l+1} \bmod \mathbf{n}_{2}^{(C)},
\end{gathered}
$$

that is, $\psi\left(\gamma_{l+1, l+1}\right)=\widehat{c}_{l+1, l+1}^{(l+1)} \gamma_{l+1, l+1} \bmod \mathbf{n}_{2}^{(C)}$. By the method of modularizing a maximal ideal of $R$ to a residue field, we know that $\tilde{a}_{j, j+1}^{(j)}, \widehat{c}_{l+1, l+1}^{(l+1)} \in R^{*}, j=1, \ldots, l$.

Lemma 4.2. Let $\psi$ be in $\operatorname{Aut}\left(\mathbf{n}_{0}^{(C)}\right)$. If $\psi\left(\alpha_{j j}\right)=\alpha_{j j} \bmod \mathbf{n}_{1}^{(C)}$, then

$$
\begin{gathered}
\psi\left(\alpha_{11}\right)=\alpha_{11}+a_{12}^{(1)} \alpha_{12} \bmod \mathbf{n}_{2}^{(C)}, \\
\psi\left(\alpha_{j j}\right)=\alpha_{j j}-a_{j-1, j}^{(j-1)} \alpha_{j-1, j}+a_{j, j+1}^{(j)} \alpha_{j, j+1} \bmod \mathbf{n}_{2}^{(C)}, \quad j=2, \ldots, l(l \geq 2), \\
\psi\left(\alpha_{l+1, l+1}\right)=\alpha_{l+1, l+1}-a_{l, l+1}^{(l)} \alpha_{l, l+1}+c_{l+1, l+1}^{(l+1)} \gamma_{l+1, l+1} \bmod \mathbf{n}_{2}^{(C)} .
\end{gathered}
$$

Proof. We express $\psi\left(\alpha_{j j}\right)$ as

$$
\psi\left(\alpha_{j j}\right)=\alpha_{j j}+\sum_{i=1}^{l} a_{i, i+1}^{(j)} \alpha_{i, i+1}+c_{l+1, l+1}^{(j)} \gamma_{l+1, l+1} \bmod \mathbf{n}_{2}^{(C)}, \quad j=1, \ldots, l+1 .
$$

From $\left[\psi\left(\alpha_{j j}\right), \psi\left(\alpha_{k k}\right)\right]=0(j \neq k)$ we have

$$
\begin{gathered}
\psi\left(\alpha_{11}\right)=\alpha_{11}+a_{12}^{(1)} \alpha_{12} \bmod \mathbf{n}_{2}^{(C)}, \\
\psi\left(\alpha_{j j}\right)=\alpha_{j j}+a_{j-1, j}^{(j)} \alpha_{j-1, j}+a_{j, j+1}^{(j)} \alpha_{j, j+1} \bmod \mathbf{n}_{2}^{(C)}, \quad j=2, \ldots, l(l \geq 2), \\
\psi\left(\alpha_{l+1, l+1}\right)=\alpha_{l+1, l+1}+a_{l, l+1}^{(l+1)} \alpha_{l, l+1}+c_{l+1, l+1}^{(l+1)} \gamma_{l+1, l+1} \bmod \mathbf{n}_{2}^{(C)}
\end{gathered}
$$

where $a_{j, j+1}^{(j)}+a_{j, j+1}^{(j+1)}=0, j=1, \ldots, l$. Lemma 4.2 is proved.

Lemma 4.3. Let $\psi$ be in $\operatorname{Aut}\left(\mathbf{n}_{0}^{(C)}\right)$. If every $\psi\left(\alpha_{j j}\right)$ is expressed as the form in Lemma 4.2, one may find an inner automorphism

$$
\theta=\prod_{j=1}^{l} \theta_{a_{j, j+1}^{(j)} \alpha_{j, j+1}} \theta_{2^{-1} c_{l+1, l+1}^{(l+1)} \gamma_{l+1, l+1}}
$$


such that

$$
\theta \psi\left(\alpha_{j j}\right)=\alpha_{j j} \bmod \mathbf{n}_{2}^{(C)}, \quad j=1, \ldots, l+1
$$

Proof. Note that $\theta_{2^{-1} c_{l+1, l+1}^{(l+1)} \gamma_{l+1, l+1}}\left(\alpha_{l+1, l+1}\right)=\alpha_{l+1, l+1}-c_{l+1, l+1}^{(l+1)} \gamma_{l+1, l+1}$. Then, by Lemma 4.2, it is not difficult to prove Lemma 4.3 .

Lemma 4.4. Let $\psi$ be in $\operatorname{Aut}\left(\mathbf{n}_{0}^{(C)}\right)$. If $\psi\left(\alpha_{j j}\right)=\alpha_{j j} \bmod \mathbf{n}_{k}^{(C)}, j=1, \ldots, l+1(1 \leq k \leq l+1)$, then

$$
\begin{aligned}
& \psi\left(\alpha_{j j}\right)=\alpha_{j j}+a_{j, j+k}^{(j)} \alpha_{j, j+k} \bmod \mathbf{n}_{k+1}^{(C)}, \quad j=1, \ldots, \min \{k, l-k+1\}(k \leq l, l \geq 1), \\
& \psi\left(\alpha_{j j}\right)=\alpha_{j j}-a_{j-k, j}^{(j-k)} \alpha_{j-k, j}+a_{j, j+k}^{(j)} \alpha_{j, j+k} \bmod \mathbf{n}_{k+1^{\prime}}^{(C)} \\
& j=k+1, \ldots, l-k+1\left(k \leq\left[\frac{l+1}{2}\right], l \geq 2\right), \\
& \psi\left(\alpha_{j j}\right)=\alpha_{j j}-a_{j-k, j}^{(j-k)} \alpha_{j-k, j}+c_{j, 2 l-k-j+3}^{(j)} \gamma_{j, 2 l-k-j+3} \bmod \mathbf{n}_{k+1}^{(C)}, \\
& j=l-k+2, \ldots, l-\left[\frac{k}{2}\right]+1\left(k \leq\left[\frac{l+1}{2}\right], l \geq 1\right), \\
& \psi\left(\alpha_{j j}\right)=\alpha_{j j}+c_{j, 2 l-k-j+3}^{(j)} \gamma_{j, 2 l-k-j+3} \bmod \mathbf{n}_{k+1}^{(C)}, \\
& j=l-k+2, \ldots, l-\left[\frac{k}{2}\right]+1\left(1+\left[\frac{l+1}{2}\right] \leq k, l \geq 2\right), \\
& \psi\left(\alpha_{j j}\right)=\alpha_{j j}+c_{j, 2 l-k-j+3}^{(j)} \gamma_{j, 2 l-k-j+3} \bmod \mathbf{n}_{k+1}^{(C)} \text {, } \\
& j=\frac{l}{2}+1\left(k=\frac{l}{2}+1, l \geq 4 \text {, here } p \text { even }\right), \\
& \psi\left(\alpha_{j j}\right)=\alpha_{j j}-a_{j-k, j}^{(j-k)} \alpha_{j-k, j}+c_{j, 2 l-k-j+3}^{(j)} \gamma_{j, 2 l-k-j+3} \bmod \mathbf{n}_{k+1}^{(C)}, \\
& j=\frac{l}{2}+2, \ldots, l-\left[\frac{k}{2}\right]+1\left(k=\frac{l}{2}+1, l \geq 4 \text {, here } p \text { even }\right), \\
& \psi\left(\alpha_{j j}\right)=\alpha_{j j}+c_{2 l-k-j+3, j}^{(2 l-k+j+3)} \gamma_{2 l-k-j+3, j} \bmod \mathbf{n}_{k+1}^{(C)}, \\
& j=l-\left[\frac{k}{2}\right]+2, \ldots, k\left(l+2 \leq k+\left[\frac{k}{2}\right], l \geq 3\right), \\
& \psi\left(\alpha_{j j}\right)=\alpha_{j j}-a_{j-k, j}^{(j-k)} \alpha_{j-k, j}+c_{2 l-k-j+3, j}^{(2 l-k-j+3)} \gamma_{2 l-k-j+3, j} \bmod \mathbf{n}_{k+1}^{(C)} \\
& j=\max \left\{k+1, l-\left[\frac{k}{2}\right]+2\right\}, \ldots, l+1\left(k \leq p,\left[\frac{k}{2}\right] \geq 1, l \geq 2\right) \text {. }
\end{aligned}
$$

Proof. We express $\psi\left(\alpha_{j j}\right), j=1, \ldots, l+1$, as

$$
\psi\left(\alpha_{j j}\right)=\alpha_{j j}+\sum_{i=1}^{l-k+1} a_{i, i+k}^{(j)} \alpha_{i, i+k}+\sum_{i=l+2-[(k+1) / 2]}^{l+1} c_{2 l-k-i+3, i}^{(j)} \gamma_{2 l-k-i+3, i} \bmod \mathbf{n}_{k+1}^{(C)} .
$$


When $k=1$ that is the case in Lemma 4.2. The conclusion follows from repeating the process of proving Lemma 4.4 .

Lemma 4.5. Let $\psi$ be in $\operatorname{Aut}\left(\mathbf{n}_{0}^{(C)}\right)$. If every $\psi\left(\alpha_{j j}\right)$ be expressed as the form in Lemma 4.4, one may find an inner automorphism

$$
\theta=\prod_{j=1}^{l-k+1} \theta_{a_{j, j+k}^{(j)}} \alpha_{j, j+k} \prod_{j=l-k+2}^{l-[k / 2]+1} \theta_{\left(1+\delta_{j l}\right)^{-1} c_{j, 2 l-k-j+3}^{(j)} \gamma_{j, 2 l-k-j+3}}
$$

where

$$
\delta_{i j}=\left\{\begin{array}{l}
1, i=j, \\
0, i \neq j,
\end{array} h=l+1-(k-1) / 2(k \text { an odd })\right.
$$

Then

$$
\theta \psi\left(\alpha_{j j}\right)=\alpha_{j j} \bmod \mathbf{n}_{k+1}^{(C)}, \quad i=1, \ldots, l+1
$$

Proof. Apply $\theta$ to $\psi\left(\alpha_{j j}\right)$ and use Lemma 4.4 to obtain Lemma 4.5.

Lemma 4.6. Let $\psi$ be in $\operatorname{Aut}\left(\mathbf{n}_{0}^{(C)}\right)$. If $\psi\left(\alpha_{j j}\right)=\alpha_{j j} \bmod \mathbf{n}_{k}^{(C)}, j=1, \ldots, l+1, l+1 \leq k \leq 2 l+1(l \geq 1)$, then

$$
\begin{gathered}
\psi\left(\alpha_{j j}\right)=\alpha_{j j}+c_{j, 2 l-k-j+3}^{(j)} \gamma_{j, 2 l-k-j+3} \bmod \mathbf{n}_{k+1}^{(C)}, \quad j=1, \ldots, l-\left[\frac{k}{2}\right]+1, \\
\psi\left(\alpha_{j j}\right)=\alpha_{j j}+c_{2 l-k-j+3, j}^{(2 l-k-j+3)} \gamma_{2 l-k-j+3, j} \bmod \mathbf{n}_{k+1}^{(C)}, \quad j=l-\left[\frac{k}{2}\right]+2, \ldots, 2 l-k+2, \\
\psi\left(\alpha_{j j}\right)=\alpha_{j j} \bmod \mathbf{n}_{k+1}^{(C)}, \quad j=2 l-k+3, \ldots, l+1 .
\end{gathered}
$$

Proof. We express $\psi\left(\alpha_{j j}\right), j=1, \ldots, l+1$, as

$$
\psi\left(\alpha_{j j}\right)=\alpha_{j j}+\sum_{i=1}^{l-[k / 2]+1} c_{i, 2 l-k-i+3}^{(j)} \gamma_{i, 2 l-k-i+3} \bmod \mathbf{n}_{k+1}^{(C)}
$$

The process of proving Lemma 4.6 is similar to Lemma 4.2.

Lemma 4.7. Let $\psi$ be in $\operatorname{Aut}\left(\mathbf{n}_{0}^{(C)}\right)$. If every $\psi\left(\alpha_{j j}\right)$ is expressed as the form in Lemma 4.6, one may find an inner automorphism

$$
\theta=\prod_{j=1}^{l-[k / 2]+1} \theta_{\left(1+\delta_{j h}\right)^{-1} c_{j, 2 l-k-j+3}^{(j)} \gamma_{j, 2 l-k-j+3}}
$$


where $h=l+1-(k-1) / 2(k$ an odd $)$. Then

$$
\theta \psi\left(\alpha_{j j}\right)=\alpha_{j j} \bmod \mathbf{n}_{k+1}^{(C)}
$$

When $k=2 l+1, \theta \psi\left(\alpha_{j j}\right)=\alpha_{j j}, j=1, \ldots, l+1$.

Proof. It is similar to proving Lemma 4.5.

Lemma 4.8. When $l \geq 1$, let $\psi$ be in $\operatorname{Aut}\left(\mathbf{n}_{0}^{(C)}\right)$. If $\psi\left(\alpha_{j j}\right)=\alpha_{j j}, j=1, \ldots, l+1$, there exists a diagonal automorphism $\lambda_{D}$ such that $\lambda_{D} \psi\left(\alpha_{j, j+1}\right)=\alpha_{j, j+1}, i=1, \ldots, l$, and $\lambda_{D} \psi\left(\gamma_{l+1, l+1}\right)=\gamma_{l+1, l+1}$.

Proof. By Lemma 4.1 we know that $\psi\left(\alpha_{j, j+1}\right)=\tilde{a}_{j, j+1}^{(j)} \alpha_{j, j+1} \bmod \mathbf{n}_{2}^{(C)}$ and $\psi\left(\gamma_{l+1, l+1}\right)=\widehat{c}_{l+1, l+1}^{(l+1)}$ $\gamma_{l+1, l+1} \bmod \mathbf{n}_{2}^{(C)}$, where $\tilde{a}_{j, j+1}^{(j)}, \widehat{c}_{l+1, l+1}^{(l+1)} \in R^{*}, j=1, \ldots, l$. We express $\psi\left(\alpha_{j, j+1}\right)$ and $\psi\left(\gamma_{l+1, l+1}\right)$, respectively, as

$$
\begin{aligned}
\psi\left(\alpha_{j, j+1}\right)= & \tilde{a}_{j, j+1}^{(j)} \alpha_{j, j+1}+\sum_{k=2}^{l} \sum_{i=1}^{l-k+1} \tilde{a}_{i, i+k}^{(j)} \alpha_{i, i+k} \\
& +\sum_{k=2}^{l} \sum_{i=l+2-[(k+1) / 2]}^{l+1} \tilde{c}_{2 l-k-i+3, i}^{(j)} \gamma_{2 l-k-i+3, i} \\
& +\sum_{k=l+1}^{2 l+1} \sum_{i=1}^{l+1-[k / 2]} \widetilde{c}_{i, 2 l-k-i+3}^{(j)} \gamma_{i, 2 l-k-i+3,} 1 \leq j \leq l, \\
\psi\left(\gamma_{l+1, l+1}\right)= & \widehat{c}_{l+1, l+1}^{(l+1)} \gamma_{l+1, l+1}+\sum_{k=2}^{l} \sum_{i=1}^{l-k+1} \widehat{a}_{i, i+k}^{(l+1)} \alpha_{i, i+k} \quad \\
& +\sum_{k=2 i=l+2-[(k+1) / 2]}^{l} \widehat{c}_{2 l-k-i+3, i}^{l+1} \gamma_{2 l-k-i+3, i}+\sum_{k=l+1}^{2 l+1} \sum_{i=1}^{l+1-[k / 2]} \widehat{c}_{i, 2 l-k-i+3}^{(l+1)} \gamma_{i, 2 l-k-i+3} .
\end{aligned}
$$

Then

$$
\begin{aligned}
\varphi\left(\alpha_{j, j+1}\right) & =\left[\varphi\left(\alpha_{j j}\right),\left[\varphi\left(\alpha_{j, j+1}\right), \varphi\left(\alpha_{j+1, j+1}\right)\right]\right] \\
& =\tilde{a}_{j, j+1}^{(j)} \alpha_{j, j+1}-\widetilde{c}_{j, j+1}^{(j)} \gamma_{j, j+1}, \quad j=1, \ldots, l .
\end{aligned}
$$

In addition,

$$
\begin{aligned}
\varphi\left(\alpha_{j, j+1}\right) & =\left[\varphi\left(\alpha_{j j}\right),\left[\left(\tilde{a}_{j, j+1}^{(j)} \alpha_{j, j+1}-\tilde{b}_{j, j+1}^{(j)} \beta_{j, j+1}\right), \varphi\left(\alpha_{j+1, j+1}\right)\right]\right] \\
& =\tilde{a}_{j, j+1}^{(j)} \alpha_{j, j+1}+\tilde{c}_{j, j+1}^{(j)} \gamma_{j, j+1}, \quad j=1, \ldots, l .
\end{aligned}
$$


Thus $\tilde{c}_{j, j+1}^{(j)}=0, j=1, \ldots, l$. So

$$
\psi\left(\alpha_{j, j+1}\right)=\tilde{a}_{j, j+1}^{(j)} \alpha_{j, j+1}, \quad j=1, \ldots, l .
$$

Furthermore,

$$
\begin{aligned}
2 \psi\left(\gamma_{l+1, l+1}\right) & =\left[\psi\left(\alpha_{l+1, l+1}\right), \psi\left(\gamma_{l+1, l+1}\right)\right] \\
& =2 \widehat{c}_{l+1, l+1}^{(l+1)} \gamma_{l+1, l+1}+\sum_{i=1}^{l-1} \widehat{a}_{i, l+1}^{(l+1)} \alpha_{i, l+1}+\sum_{i=1}^{l-1} \widehat{c}_{i, l+1}^{(l+1)} \gamma_{i, l+1} .
\end{aligned}
$$

From $\left[\psi\left(\alpha_{i i}\right), \psi\left(\gamma_{l+1, l+1}\right)\right]=0(i \neq l-1)$, we have $\widehat{a}_{i, l+1}^{(l+1)}=0$ and $\widehat{c}_{i, l+1}^{(l+1)}=0, i=1, \cdots, l-1$, that is,

$$
\psi\left(\gamma_{l+1, l+1}\right)=\widehat{c}_{l+1, l+1}^{(l+1)} \gamma_{l+1, l+1}
$$

Let $d=\operatorname{diag}\left(d_{1}, \ldots, d_{l+1}\right)$ and $d_{0}=\widehat{c}_{l+1, l+1}^{(l+1)} d_{l+1}^{2}$, where $d_{1}=1, d_{j}=\prod_{i=2}^{j} \tilde{a}_{j-i+1, j-i+2}^{(j-i+1)}, j=2, \ldots, l+1$. Applying $\lambda_{D}$ to $\psi\left(\alpha_{j, j+1}\right), j=1, \ldots, l$, and $\psi\left(\gamma_{l+1, l+1}\right)$, we get the result.

\section{Proofs of Main Results}

Proof of Theorem 1.1. By Lemmas 4.3, 4.5, 4.7, and 4.8 we have $\lambda_{D} \theta \psi\left(\alpha_{j j}\right)=\alpha_{j j}, j=1, \ldots, l+1$, $\lambda_{D} \theta \psi\left(\alpha_{j, j+1}\right)=\alpha_{j, j+1}, j=1, \ldots, l$, and $\lambda_{D} \theta \psi\left(\gamma_{l+1, l+1}\right)=\gamma_{l+1, l+1}$. Since the set $\left\{\gamma_{l+1, l+1}, \alpha_{l+1, l+1}, \alpha_{j j}\right.$, $\left.\alpha_{j, j+1} \mid j=1, \ldots, l\right\}$ generates $\mathbf{t}_{l+1}^{(C)}(R)$, we know that $\lambda_{D} \theta \psi$ is the identity automorphism of $\mathbf{t}_{l+1}^{(C)}(R)$. Hence $\psi=\theta^{\prime} \lambda_{D^{-1}}$. The uniqueness of the decomposition follows from Theorem 1.2.

Proof of Theorem 1.2. By the first part of Theorem 1.1 we have Aut $\left(\mathbf{t}_{l+1}^{(C)}(R)\right)=\supset \Phi$. For any $x \in$ $\mathbf{t}_{l+1}^{(C)}(R)$ and $\alpha_{i j} \in \mathbf{n}_{1}^{(C)}$ we have

$$
\begin{aligned}
\lambda_{D} \theta_{a \alpha_{i j}} \lambda_{D}^{-1}(x) & =D\left(I_{n}+a \alpha_{i j}\right) D^{-1} x D\left(I_{n}+a \alpha_{i j}\right)^{-1} D^{-1} \\
& =\left(I_{n}+a \lambda_{D}\left(\alpha_{i j}\right)\right) x\left(I_{n}+a \lambda_{D}\left(\alpha_{i j}\right)\right)^{-1} \\
& =\theta_{a \lambda_{D}\left(\alpha_{i j}\right)}(x) .
\end{aligned}
$$

So $\lambda_{D} \theta_{a \alpha_{i j}}=\theta_{a \lambda_{D}\left(\alpha_{i j}\right)} \lambda_{D}$. For $\gamma_{i j} \in \mathbf{n}_{1}^{(C)}$, we have $\lambda_{D} \theta_{c \gamma_{i j}}=\theta_{c \lambda_{D}\left(\gamma_{i j}\right)} \lambda_{D}$. Therefore, $\supset \triangleleft \supset \boldsymbol{\Phi}$. Obviously $\supset \cap \Phi=1$. Then, $\supset \boldsymbol{\Phi}=\supset \ltimes \Phi$.

\section{Discussion for $l=0$}

In this case, $\mathbf{t}_{1}^{(C)}(R)$ is generated by $\alpha_{11}$ and $\gamma_{11}$. For any automorphism $\psi$ of $\mathbf{t}_{1}^{(C)}(R)$, write $\psi\left(\alpha_{11}\right)$ and $\psi\left(\gamma_{11}\right)$, respectively, as $\psi\left(\alpha_{11}\right)=a_{11} \alpha_{11}+c_{11} \gamma_{11}$ and $\psi\left(\gamma_{11}\right)=c \gamma_{11}$, where $c \in$ $R^{*}$. From $2 \psi\left(\gamma_{11}\right)=\left[\psi\left(\alpha_{11}\right), \psi\left(\gamma_{11}\right)\right]$, we have $a_{11}=1$. Then $\theta_{2^{-1} c_{11} \gamma_{11}} \psi\left(\alpha_{11}\right)=\alpha_{11}$ and $\theta_{2^{-1} c_{11} \gamma_{11}} \psi\left(\gamma_{11}\right)=c \gamma_{11}$. Also $\eta_{c} \theta_{2^{-1} c_{11} \gamma_{11}} \psi\left(\alpha_{11}\right)=\alpha_{11}$ and $\eta_{c} \theta_{2^{-1} c_{11} \gamma_{11}} \psi\left(\gamma_{11}\right)=\gamma_{11}$. So $\psi=\theta_{-2^{-1} c_{11} \gamma_{11}}$ $\eta_{c^{-1}}$. 


\section{Acknowledgments}

The research was supported partially by National Natural Science Foundation of China (Grant nos. 10871056 and 10971150) and by Science Research Foundation in Harbin Institute of Technology (Grant no. HITC200708).

\section{References}

[1] G. Ancochea, "On semi-automorphisms of division algebras," Annals of Mathematics. Second Series, vol. 48, pp. 147-153, 1947.

[2] K. I. Beidar, M. Brešar, and M. A. Chebotar, “Jordan isomorphisms of triangular matrix algebras over a connected commutative ring," Linear Algebra and Its Applications, vol. 312, no. 1-3, pp. 197-201, 2000.

[3] M. Brešar, "Jordan mappings of semiprime rings," Journal of Algebra, vol. 127, no. 1, pp. 218-228, 1989.

[4] Y. A. Cao, "Automorphisms of the Lie algebra of strictly upper triangular matrices over certain commutative rings," Linear Algebra and its Applications, vol. 329, no. 1-3, pp. 175-187, 2001.

[5] L. N. Herstein, "Jordan homomorphisms," Transactions of the American Mathematical Society, vol. 81, pp. 331-351, 1956.

[6] S. Jøndrup, "Automorphisms of upper triangular matrix rings," Archiv der Mathematik, vol. 49, no. 6, pp. 497-502, 1987.

[7] F. Kuzucuoglu and V. M. Levchuk, "The automorphism group of certain radical matrix rings," Journal of Algebra, vol. 243, no. 2, pp. 473-485, 2001.

[8] X. T. Wang and Y. M. Li, "Decomposition of Jordan automorphisms of triangular matrix algebra over commutative rings," Glasgow Mathematical Journal, vol. 52, no. 3, pp. 529-536, 2010.

[9] D. Ž. Doković, "Automorphisms of the Lie algebra of upper triangular matrices over a connected commutative ring," Journal of Algebra, vol. 170, no. 1, pp. 101-110, 1994.

[10] Y. A. Cao, "Automorphisms of certain Lie algebras of upper triangular matrices over a commutative ring," Journal of Algebra, vol. 189, no. 2, pp. 506-513, 1997.

[11] X. T. Wang and H. You, "Decomposition of Lie automorphisms of upper triangular matrix algebra over commutative rings," Linear Algebra and its Applications, vol. 419, no. 2-3, pp. 466-474, 2006. 


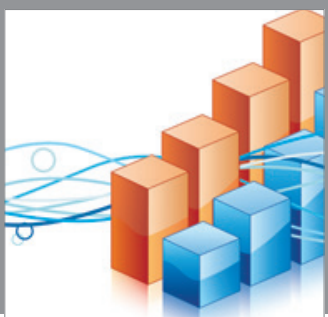

Advances in

Operations Research

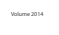

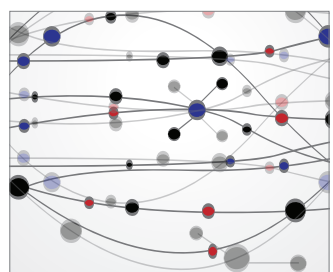

\section{The Scientific} World Journal
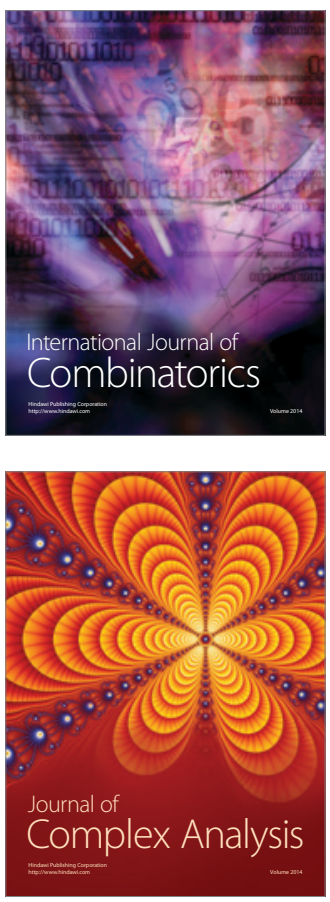

International Journal of

Mathematics and

Mathematical

Sciences
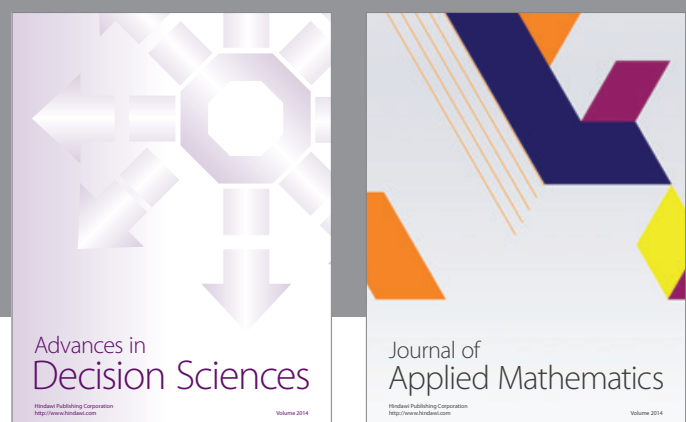

Journal of

Applied Mathematics
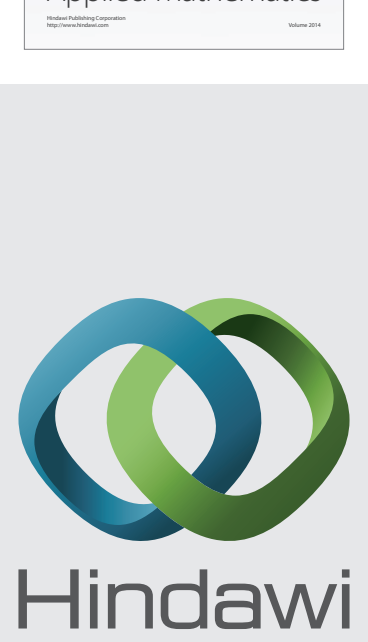

Submit your manuscripts at http://www.hindawi.com
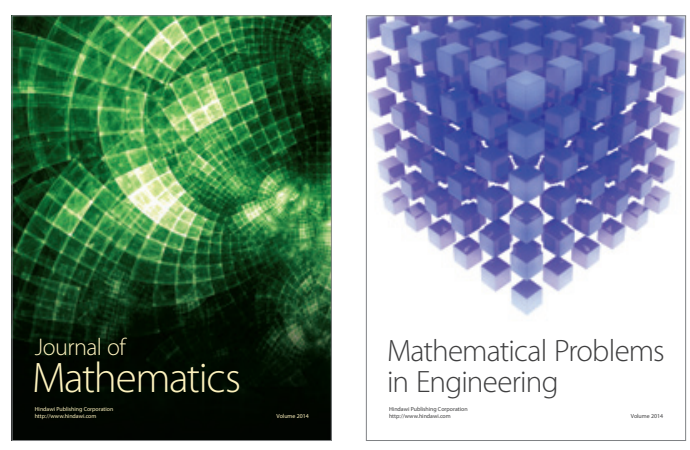

Mathematical Problems in Engineering
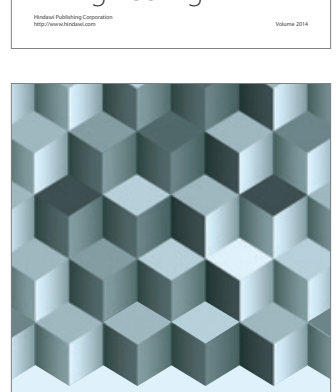

Journal of

Function Spaces
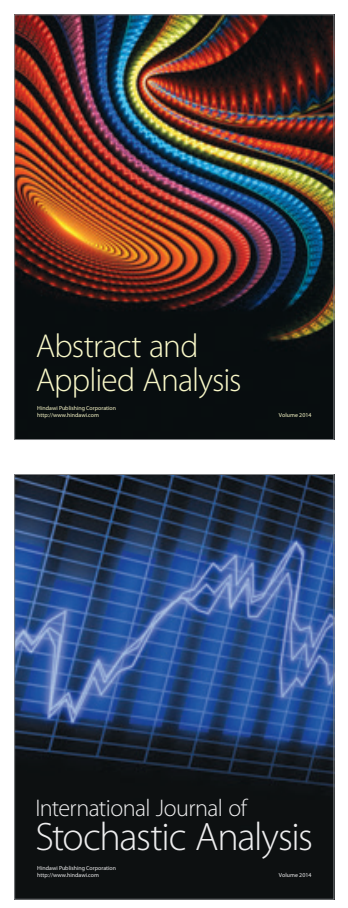

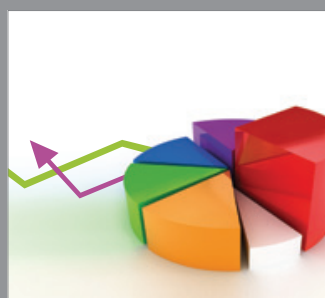

ournal of

Probability and Statistics

Promensencen
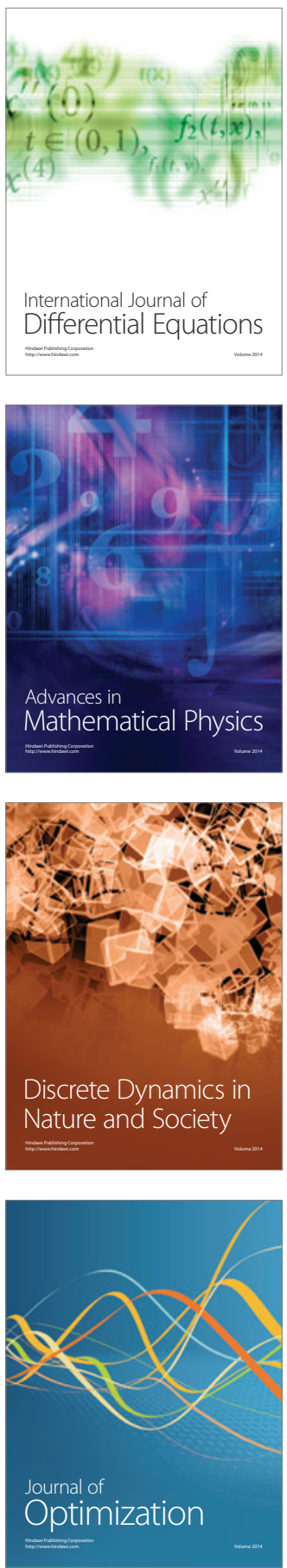